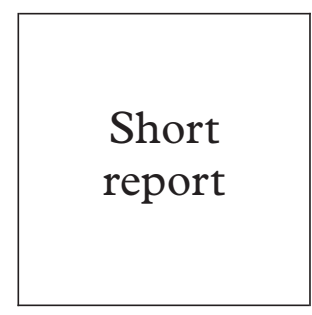
Katherine Perkins, Lori Saunders, Paul Young

\section{$\operatorname{sex}$}

\title{
An investigation of condom use by frequency of
}

Mark Williams, Michael W Ross, Anne M Bowen, Sandra Timpson, H Virginia McCoy,

Objectives: To examine the association between condom use and frequency of sex across sexual partner types.

Methods: Data were collected from African American drug users residing in Washington, DC. Participants were asked to provide the first name of the last three partners with whom they had had vaginal sex, to define the type of relationship, the number of times they had had vaginal sex in the last 30 days with each partner, and whether they had used a condom during their last sexual encounter with each partner. Condom use was examined by frequency of sex in the past 30 days controlling for partner type.

Results: Frequency of sexual encounters varied by partner type. The largest number of sexual encounters was with casual partners, followed by commercial and primary partners, respectively. Within partner types, participants had sex most frequently with primary partners. Condom use was lowest within primary partner relationships. Regardless of partner type, condom use varied consistently depending on the frequency of sex with the same partner.

Conclusions: Condom use is related to the frequency of sex between partners when partner type is controlled. The association between frequency of sex and condom use is independent of partner type, suggesting that partner type may become less influential in determining condom use as the frequency of sex increases. This finding suggests that contextual factors determining condom use go beyond partner type designations and include other relationship variables.

(Sex Transm Inf 2001;77:433-435)

Keywords: HIV; sexual transmission; drug users; condom use

School of Public

Health, University of

Texas Health Science

Center at Houston,

7000 Fannin, Houston,

TX 77030, USA

M Williams

M W Ross

K Perkins

Department of Psychology, University of Wyoming, Laramie, WY 82071, USA

A M Bowen

Department of Public Health, Florida International

University, North

Miami Campus, North

Miami, FL 33181, USA

H V McCoy

Nova Research

Company, 4600

East-West Highway,

Suite 700, Bethesda,

MD 20814, USA

S Timpson

L Saunders

P Young

Correspondence to:

Dr Mark Williams, Center

for Health Promotion and

Prevention Research, School

of Public Health, University

of Texas-Houston, 7000

Fannin, Suite 2516,

Houston, TX 77030, USA

mwilliams@sph.uth.tmc.edu

Accepted for publication 30 August 2001
Research on men who have sex with men and drug users shows higher rates of condom use during anal/vaginal sex with casual partners and lower rates with primary partners. ${ }^{1-8}$ Consistently lower rates of condom use between primary partners across different groups has led to speculation that condom use is not the result of either an objective appraisal of the risk presented by the partner or the motivation to use condoms with that partner. Condom use, particularly between primary partners, might be determined by the context of the relationship, especially feelings towards the partner and/or ideals about primary partners. ${ }^{6-13}$

The labelling of partners by type is a socially constructed variable that presumably reflects the level of attachment between partners. Partner type may neither adequately measure the level of attachment nor other contextual factors within a sexual relationship that influence condom use. Another contextual factor that may influence condom use is the frequency a couple has sex. The purpose of this study is to examine the hypothesis that condom use changes as the frequency of sex between partners changes, regardless of partner type. The focus of this analysis is the frequency of vaginal sex between the same partners in the past 30 days.

\section{Methods}

Data were collected between January 1997 and November 1997 in Washington, DC, using the sexual risk reduction questionnaire (SRQ). The questionnaire was designed to measure the psychosocial determinants of condom use and condom use behaviours in treatment drug users and to collect demographic, drug use, and sexual behaviour data. Measures in the SRQ have been shown to be both valid and reliable with this and similar populations. ${ }^{14-21}$ Candidates for the study were recruited using targeted sampling. ${ }^{22}{ }^{23}$ Individuals selected to take part were 18 years of age and over; were African American; self identified as heterosexual; had smoked crack cocaine in the 48 hours before being interviewed; had had vaginal sex at least once in the 7 days before being screened for the study; and were willing to sign a written informed consent form. Participants' reports of cocaine use in the 48 hours before the screening were confirmed by urinalysis. A trained research assistant interviewed participants privately in a store front office. Independent committees for the protection of human subjects reviewed and approved study procedures.

Participants were asked to recall the last three partners with whom they had had sex, whether condoms were used, and to describe their relationships with those partners. For this analysis, condom use is the dependent variable. Condom use was measured as condom use the last time the partners had vaginal sex and recorded as a bivariate response. The independent variables for this analysis were partner type and the frequency the respondent had sex with the same partner. Partner type was measured by asking participants to choose 


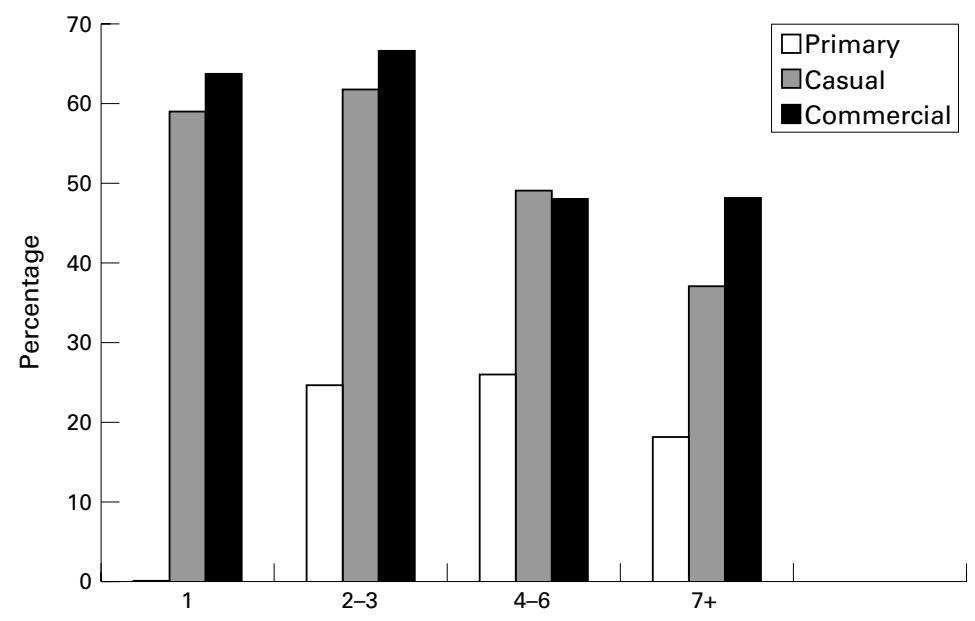

Figure 1 Condom use by the frequency of sex controlling for partner type.

from a list the descriptor that best characterised the partner. Frequency of sex was measured as the number of times the participant had vaginal sex with the same, identified partner in the 30 days before being interviewed. Responses were recorded using a categorical measure ranging from zero to seven or more times. Responses of zero, signifying no sex between the partners in the previous 30 days, were dropped from the analysis.

\section{Results}

One hundred and ninety nine individuals, all of whom were African American, took part in the study. Of these, $103(52 \%)$ were male. Age ranged from 22 to 61 years, with an average of 36.6 years (median 36 ). One hundred and fifty eight participants $(80 \%)$ described themselves as single. Two thirds of the sample $(66 \%)$ reported living in someone else's home and about a quarter $(24 \%)$ were living in their own house or apartment. Most (41\%) reported having a high school education or its equivalent, although a third (33\%) reported less than a high school education. Slightly more than a quarter $(26 \%)$ reported having some college education. Owing to eligibility requirements, all participants had smoked crack cocaine in the previous 30 days. The number of female sex partners reported by men in the 30 days before the interview ranged from 1 to 30 partners, with an average of 4.1 (median 3) partners. The number of male partners reported by women ranged from 1 to 350 partners, with an average of 32.0 (median 10) partners. The higher number of sex partners reported by women is related to trading sex for money and/or drugs.

Because of eligibility requirements limiting participation to drug users who had vaginal sex in the 7 days before screening, all 199 participants described at least one sexual encounter with a partner. Of these, $178(89 \%)$ described an encounter with a second partner and $168(84 \%)$ with a third partner. If every participant had reported having had vaginal sex with three partners in the previous 30 days, the maximum number of sexual encounters possible is 597. Participants reported a total of
492 sexual encounters, or $82 \%$ of the maximum possible. Of these, most sexual encounters $(41 \%)$ were between casual partners; $37 \%$ of sexual encounters were between commercial partners and $21 \%$ between primary partners.

Frequency of sex with the same partner is related to partner type $\left(\chi^{2}=147.9, \mathrm{p}<0.001\right)$. Over two thirds of those having sex with a primary partner had sex with that partner seven or more times. Thirty nine per cent of those describing a last sexual encounter with a casual partner reported having sex with that partner two to three times. More than a quarter $(27 \%)$ reported having sex with the same casual partner seven or more times. Sex with the same commercial partner was less frequent. Almost half of the participants describing a last sexual encounter with a commercial partner reported having had sex with the same partner once. Only $13 \%$ of those reporting sex with a commercial partner had sex seven or more times with the same partner.

As expected, condom use varied significantly by partner type $\left(\chi^{2}=46.09, \mathrm{p}<0.000\right)$. Participants used condoms $20 \%$ of the time during encounters with primary partners, $52 \%$ with casual partners, and $61 \%$ with commercial partners. As hypothesised, condom use within partner type varied as the frequency of sex between partners increased. Within partner type, the association appears to be curvilinear, as shown in figure 1 , and differences are statistically significant $(p<0.000)$. The clearest pattern of condom use by frequency is evident for sex between casual partners. Condom use during the last sexual encounter with a casual partner is highest if the participant reported having had sex with the same partner two to three times. Condom use is lowest if the participant reported having had sex with the same partner seven or more times. Similarly, condom use during encounters with commercial partners is highest at three to four encounters and lower after four to six encounters.

\section{Discussion}

Our data demonstrate that frequency of sex is a key determinant for condom use in specific sexual partnerships. Data used in the analysis were collected in 1997. However, we are aware of no research suggesting that these data are any less useful for describing the sexual behaviours of drug users now than when they were collected. The study is limited by the use of targeted sampling. Although appropriate and valid when sampling hidden and elusive populations, targeted sampling does not generate a response rate. Thus, the number of refusals is unknowable. ${ }^{24}$ The study relies on self report of sexual relationships and behaviours. ${ }^{17}$ This limitation was addressed by restricting questions about sexual behaviours to the last three partners and to sex in the last 30 days. Using this strategy, reports by drug users of their sexual behaviours are reliable and valid. ${ }^{18-20}$ Even so, self reports of sexual behaviours are subject to recall bias and are difficult to verify. The cross sectional design of the study limits the ability to draw causal inferences. While we can show an association between frequency of 
sex between partners and condom use, we cannot establish causality. A further limitation is the number of cases within partner type categories, particularly in the low frequency categories. Our findings are also limited because no data were gathered on condom use during each sexual encounter mentioned in the previous 30 days.

Previous research has established that there are distinct differences in condom use by partner type. ${ }^{1-8}$ We hypothesised that frequency of sex with the same partner would affect condom use, and our data support this hypothesis. Condom use, while exhibiting variations in the level of condom use by partner type, presents a pattern of use related to the frequency of sex between partners. Given the trajectory of the association between condom use and frequency, if more data points had been available we expect that condom use would continue to decline as the frequency of sex between partners increases. The association between frequency of sex and condom use is independent of partner type, suggesting a more complex contextual structure in determining condom use that goes beyond partner type designations.

The opinions expressed here are solely those of the authors. Support for this research was provided by a grant from the National Institute on Drug Abuse. The authors thank the National Institute on Drug Abuse. The authors thank the
anonymous reviewers who examined previous versions of this research.

1 Manderson L, Tye L, Rajanayagam K. Condom use in heterosexual sex: a review of research. In Catalan J, Sherr L erosexual sex: a review of research. In Catalan J, Sherr L,
Hedge $\mathrm{B}$, eds. The impact of AIDS. Psychological and social Hedge B, eds. The impact of AIDS. Psychological and social aspects of HIV infection. Am

2 Sheeran, P, Abraham C, Orbell S. Psychosocial correlates of heterosexual condom use: a meta-analysis. Psychol Bull 1999;125: 90-132.

3 Silvestre A, Lyter D, Valdiserri R, et al. Factors related to seroconversion among homosexual and bisexual men afte attending a risk education session. AIDS 1989;3:147-50.

$4 \mathrm{Ku} \mathrm{L}$, Sonnenstein F, Pleck, J. The dynamics of young men' condom use during and across relationships. Family Planning Perspectives 1994;26:246-51.
5 Hays R, Kegeles S, Coates T. Unprotected sex and HIV risk taking among young gay men within boyfriend relationtaking among young gay men with
ships. AIDS Ed Prev 1997;9:314-29.

6 Williams M, Bowen A, Elwood W, et al. An elicitation of the determinants of condom use among African Americans who smoke crack cocaine. Cult, Health, Sexual 2000;2:1532.

7 Ross M, Wodak A, Gold J. Sexual behaviour in injecting drug users. F Psych Hum Sexual 1992;5:89-104.

8 Ross M, Wodak A, Miller M, et al. Sexual partner choice in injecting drug users from a "critical incident" measure: its implications for assessing risk of spread. Sexological Revue 1993;1:77-92.

9 Williams S, Kimble D, Covell N, et al. College students use mplicit personality theory instead of safer sex. f Appl Soc Psych 1992;22:921-33.

10 Gold R, Skinner M, Ross M. Unprotected anal intercourse in HIV-infected and non-HIV-infected gay men. f Sex Res 1994;31:59-77.

11 Buchanan D, Poppen P, Reisen C. The nature of partner relationship and AIDS sexual risk-taking in gay men. Psychol Health 1996;11:541-55.

12 Remien R, Carballo-Diéguez A, Wagner G. Intimacy and sexual risk behavior in serodiscordant male couples. AIDS Care 1995;7:429-38

13 Inciardi J, Lockwood D, Pottieger A. Women and crackcocaine. New York: Macmillan, 1993

14 McCoy C, Inciardi J. Sex, drugs, and continuing spread of AIDS. Los Angeles: Roxbury Publishing Company, 1995

15 Weatherby N, Bowen A, McCoy C, et al. Unpublished test/ retest data, 1998.

16 Catania J, Gibson D, Marin B, et al. Response bias in assessing sexual behaviors relevant to HIV transmission. Eval Prog Planning 1990;13:19-29.

17 Weatherby N, Needle R, Cesari H, et al. Validity of self-reported drug use among injection drug users and crack cocaine users recruited through street outreach. Eval Prog Planning 1994;17:347-55.

18 Dowling-Guyer S, Johnson M, Fisher D, et al. Reliability of drug users' self-reported HIV risk behaviors and validity of self-reported recent drug use. Assessment 1994;1:383-92.

19 Needle R, Fisher D, Weatherby N, et al. The reliability of self-reported HIV risk behaviors of drug users. Psychiatr Addict Behav 1995;9:242-50.

20 Aral S, Peterman R. Measuring outcomes of behavioural interventions for STD/HIV prevention. Int $\mathcal{F}$ STD AIDS 1996;7:30-8.

21 Darke S. Self-report among injecting drug users: a review. Drug Alcohol Depend 1998;51:253-63.

22 Watters J, Biernacki P. Targeted sampling: options for the study of hidden populations. Soc Problems 1989;36:416-30.

23 Carlson R, Wang J, Siegal $\mathrm{H}$, et al. An ethnographic approach to targeted sampling and solution in AIDS prevention research among injection drug and crackcocaine users. Human Org 1994;53:279-86.

24 Stueve A, O'Donnell L, Duran R, et al. Time-space sampling in minority communities: results with young Latino men who have sex with men. Am $f$ Public Health 2001;91:922-6. 